Aunque la transmisión por roedores es la ruta más frecuente de infección por hantavirus, frente a cualquier caso nuevo de síndrome pulmonar por hantavirus que no manifieste haber tenido contacto con roedores se debe explorar la posibilidad de infección interpersonal. Para ello se debe buscar un vínculo epidemiológico con algún caso previo, confirmar la homología de las secuencias nucleotídicas de ambos casos y descartar la posibilidad de que el paciente se haya infectado mediante el contacto con un roedor que tenga la misma homología nucleotídica del caso índice sospechado. (Martinez VP, et al. Person-to-person transmission of Andes virus. Emerg Infect Dis [publicación periódica en línea]. 2005;11(12). Hallado en: http://www.cdc.gov/ ncidod/EID/vol11no12/05-0501.htm. Acceso el 1 de diciembre de 2005).

\section{Efecto de la transición epidemiológica en los gastos de salud}

Las autoridades sanitarias de América Latina buscan alternativas para reducir el alto costo de los servicios de salud durante el período de transición epidemiológica, ya que además de crecer la demanda, aumentará el costo de esos servicios.

El objetivo de este estudio longitudinal fue calcular el costo y los recursos financieros necesarios para atender los casos de hipertensión arterial y diabetes tipo 2 en el período comprendido entre 2004 y 2006 en México. La demanda anual y el costo directo de los servicios de salud para ambas enfermedades se calculó a partir del número de casos tratados por la Secretaría de Salud (SSA), el Instituto Mexicano del Seguro Social (IMSS) y el Instituto de Seguro Social y Servicios de los Trabajadores del Estado (ISSSTE), ajustados según el tipo de institución.

El costo de la atención sanitaria calculado para un paciente con diabetes fue de US\$ 613,00 a US\$ 887,00, mientras que para un paciente con hipertensión arterial fue de US\$ 485,00 a US\$ 622,00. Se prevé que en el año 2006, el número de casos de ambas enfermedades será mucho mayor que en 2004 y que aumentará el costo de los servicios de hospitalización.
Al IMSS le correspondieron el mayor costo directo promedio por caso y el mayor impacto económico por la atención de los pacientes con diabetes o hipertensión arterial en el período estudiado. Los costos indirectos fueron similares en las tres instituciones y sobrepasaron el costo directo total en $23 \%$. La demanda financiera para la atención ambulatoria y hospitalaria de ambas enfermedades representó el 9,5\% del presupuesto total asignado a la población sin cobertura de seguro de salud y el 13,5\% del destinado a la población asegurada.

El gasto por la atención de los pacientes con diabetes en el IMSS fue similar al del SSA y mucho mayor que el necesario para atender a los pacientes con seguro de salud en el ISSSTE.

El gasto promedio en salud de las personas de bajos ingresos que no tenían seguro (40\% de los mexicanos) representó el 15\% del gasto total en los últimos años, mientras que para la población con seguro de salud ( $50 \%$ de los mexicanos) fue el $43 \%$. El incremento relativo en el costo de la atención sanitaria de la población sin seguro de salud será $17 \%$ mayor con respecto a las personas sin seguro $(P<0,05)$. Según estos resultados, a la población asegurada le corresponderá el $75 \%$ del gasto por la atención sanitaria de los casos de diabetes e hipertensión arterial, mientras que a las personas sin seguro les corresponderá el 25\% restante.

Si los factores de riesgo y los diferentes modelos de atención sanitaria no varían hasta el año 2006, el impacto económico de los cambios epidemiológicos esperados será elevado. Otro elemento que se debe tener en cuenta es la posible competencia por los recursos entre los principales proveedores de servicios de salud y entre los programas específicos para el tratamiento de la hipertensión, la diabetes y otras enfermedades crónicas o infecciosas.

La población sin seguro de salud será la más afectada, ya que la presión de la inequidad en la distribución de los recursos se sumará a la mayor demanda de servicios de los pacientes con enfermedades crónicas o degenerativas. (Arredondo A, et al. Health care costs and financial consequences of epidemiological changes in chronic diseases in Latin America: evidence from Mexico. Public Health. 2005;119:711-20). 